\title{
Development of Electronic Transparent Communication Boards Using Two Tablets
}

\author{
Tomoya Miyasaka, Keiko Kawashima, Mamiko Kamoshida, Tadafumi Saga, Toshiaki Tanaka
}

\begin{abstract}
Neuromuscular diseases such as amyotrophic lateral sclerosis (ALS) progressively decrease voluntary motor function, gradually causing communication difficulties. Despite disease progression, a transparent communication board can be used for an extended period as one of the means of communication. It is a transparent plate on which characters including letters, numbers, and symbols are displayed, for caregivers to read intentions of patients. Nonetheless, conventional transparent communication boards have the following problems: 1 . the caregiver needs to position each character on the board so that the target character can be aligned linearly between the caregiver's eyes and the patient's eyes; 2. caregivers need to memorize or record words and sentences, because intentions of a patient are read on a character basis; and 3. the arrangement of characters on the board is fixed. The objective of this study was to develop an electronic transparent communication board (device) to solve the problems of conventional transparent communication boards and evaluate and verify its effect. The device included two tablets that were connected with each other via Wi-Fi so that the caregiver could confirm the voluntary movements of the patient by the video images taken by the attached cameras and enter the characters on the screen. Among the three problems of the conventional transparent communication board, the adjustment of the location of the transparent communication board seemed to become easier, because the device can be operated without aligning the visual line of the caregiver with that of the patient. It took longer to operate the prepared device than the conventional transparent communication board, but it is expected that the negative impacts caused by the delays can be reduced, if the communication speed and tablet performance are improved. Characters were considered to become entered more easily with the prepared system than with the approaches depending on memories and notes.
\end{abstract}

Index Terms - amyotrophic lateral sclerosis, communication assistance, transparent communication boards

\section{INTRODUCTION}

Neuromuscular diseases such as amyotrophic lateral sclerosis (ALS) [1] progressively decrease voluntary motor function, gradually causing communication difficulties [2].

This work was supported by JSPS KAKENHI Grant Number JP 26350668

Tomoya Miyasaka, Department of Physical Therapy, Faculty of Health Sciences, Hokkaido University of Science, Sapporo, Hokkaido, Japan

Keiko Kawashima, Department of Physical Therapy, Faculty of Health Sciences, Hokkaido University of Science, Sapporo, Hokkaido, Japan

Mamiko Kamoshida, Department of Physical Therapy, Faculty of Health Sciences, Hokkaido University of Science, Sapporo, Hokkaido, Japan

Tadafumi Saga, Development Department, Kuki Factory, CHINO Corporation, Saitama, Japan

Toshiaki Tanaka, Institute of Gerontology, The University of Tokyo, Tokyo, Japan, Department of Physical Therapy, Faculty of Health Sciences, Hokkaido University of Science, Sapporo, Hokkaido, Japan
There are devices that assist communication [3], but it takes time to introduce such device due to difficulties in handling with caregivers. There are many cases in which patients become unable to use the device shortly after it is introduced because of the progression of the disease.

Although communication devices using cerebral blood flow and/or brain waves as input sources have recently been developed [4], such these devices have problems: for example, they cannot be used on a daily basis due to the formation of ulcers on the skin when electrodes are attached to the skin around the face for a prolonged period, and their response time of detection is slow.

On the other hand, despite disease progression, a transparent communication board (Figure 1) [5] can be used for an extended period as one of the means of communication. It is a transparent plate on which characters including letters, numbers, and symbols are displayed, for caregivers to read intentions of patients. It can be used as long as the intention expressed by the voluntary movement of a patient can be confirmed by visual observation of a caregiver.

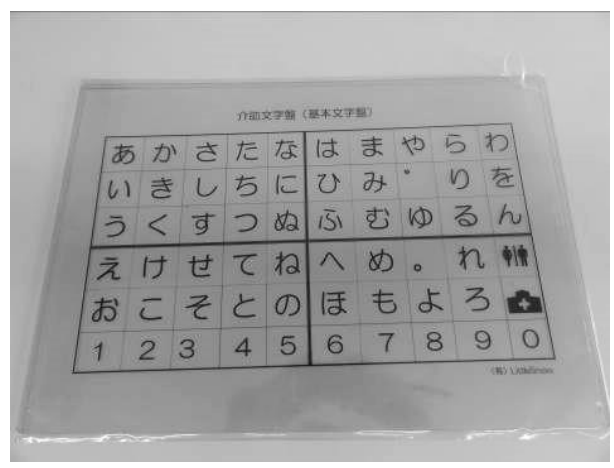

Fig. 1 Transparent communication board

Nonetheless, conventional transparent communication boards have the following problems: 1 . the caregiver needs to position each character on the board so that the target character can be aligned linearly between the caregiver's eyes and the patient's eyes; 2 . caregivers need to memorize or record words and sentences, because intentions of a patient are read on a character basis; and 3. the arrangement of characters on the board is fixed.

Researchers have provided support for communication between ALS patients and caregivers [6], [7]. Patients have requested to take the initiative in expressing their intentions, while caregivers requested to reduce the burden of assistance in communication.

The objective of this study was to develop an electronic transparent communication board (device) to solve the problems of conventional transparent communication boards and evaluate and verify its effect. The study aimed to develop a device that meets the needs of both patients and caregivers. 


\section{METHOD}

The device was created by examining the specifications of the device as well as the configuration of its hardware and application. The operation of the prepared device was tested, and its functionality was evaluated. This study was conducted in accordance with the Application No. 191 issued by the Ethics Committee of Hokkaido University of Science.

\section{A. Device Specifications}

The device included two tablets that were connected with each other via Wi-Fi so that the caregiver could confirm the voluntary movements of the patient by the video images taken by the attached cameras and enter the characters on the screen. In addition, entered words and sentences were saved to make them available for future use.

\section{B. Configuration of hardware and application}

For hardware, a pair of 10.1-inch Android tablets (Experia Z2, SONY) was used. For applications, three types of applications were used: a keyboard application (referred to as app), an input decision app, and a text input app.

\section{RESULTS}

The operation of the prepared device was confirmed, and its functionality was evaluated.

\section{A. Confirmation of operation}

The prepared device was shown in Figure 2. The left image showed the tablet connected to the communication board for the caregiver, while the right image showed the one for the patient.

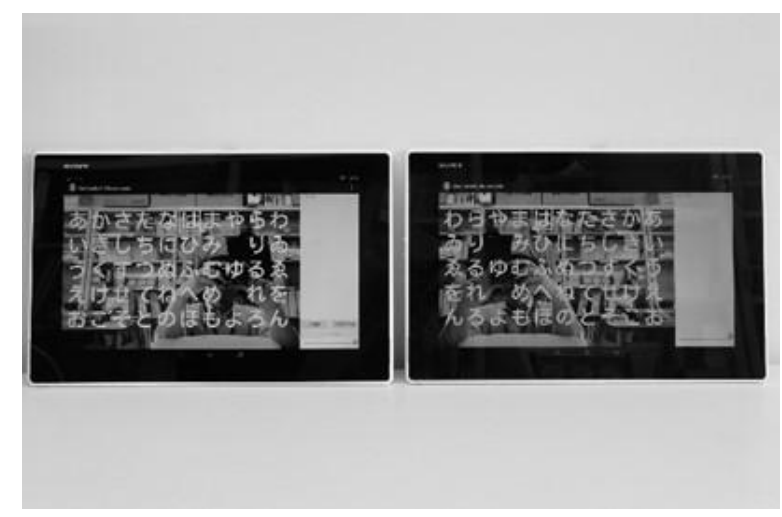

Fig. 2 Device configuration

The two tablets were connected through $\mathrm{Wi}-\mathrm{Fi}$ on a one-to-one basis and shared the communication board and text characters entered. Video images were sent only from a patient to a caregiver in one direction, and the face of the patient appeared on the screen of the tablets for both the patient and the caregiver. On the left side of the tablet screen, the character keyboard was displayed by the keyboard app. On the right side of the tablet screen, the text input application which converts the input characters into text data was displayed. When the caregiver turned the tablet to capture the patient's eyes on a character on the screen, the eyes of the patient were reflected on the same character frames on both screens of the two tablets.

The operation of the device was confirmed by using healthy adults as subjects (with a caregiver role or a patient role).

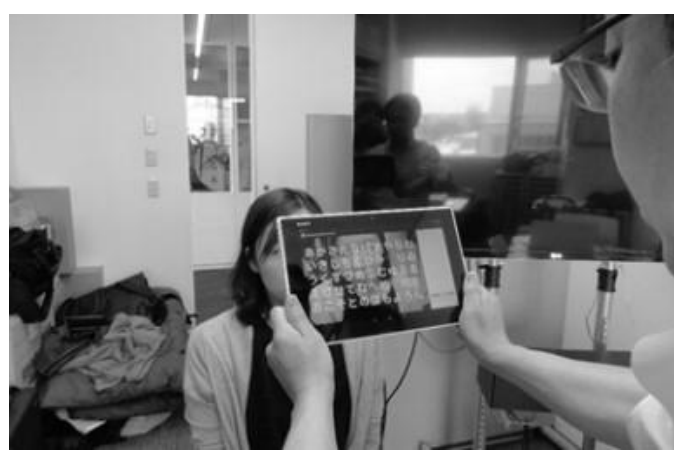

Fig. 3 System operation (caregiver side)

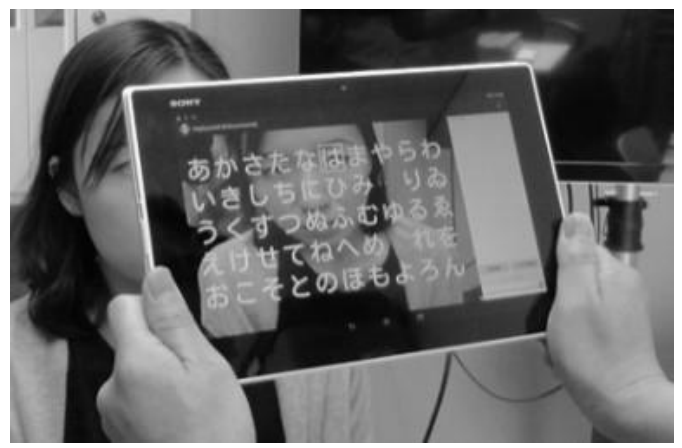

Fig. 4 Tablet (caregiver side)

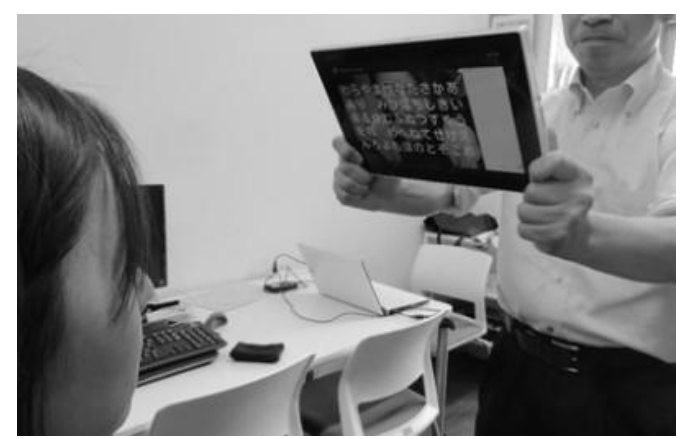

Fig. 5 System operation (patient side)

Figure 3 showed the operation status from the caregiver side. Figure 4 showed an enlarged view of the tablet on the caregiver side. Figure 5 showed the operation status from the patient side.

In the procedure, the patient and the caregiver faced each other, and the caregiver held two tablets stacked back-to-back with both hands between the two people. The caregiver adjusted the positions of the eyes of the patient in the video image to the corresponding characters of the communication board. The caregiver moved the tablet toward the left side while producing the sound of a first character of each row starting from the row of "a" (Figure 6) so that the eyes of the patient can be displayed on the corresponding character frames. Then the caregiver fixed each line number, when the patient indicated his/her intention by moving muscles including the facial expression muscle. Next, the caregiver 
moved the tablet upward while producing the sound of each character of each column, and fixed the corresponding character when the patient indicated his/her intention. Subsequently, the caregiver entered the fixed characters into the text input app by tapping characters on the screen. Words and sentences were entered by repeating the procedure described above.

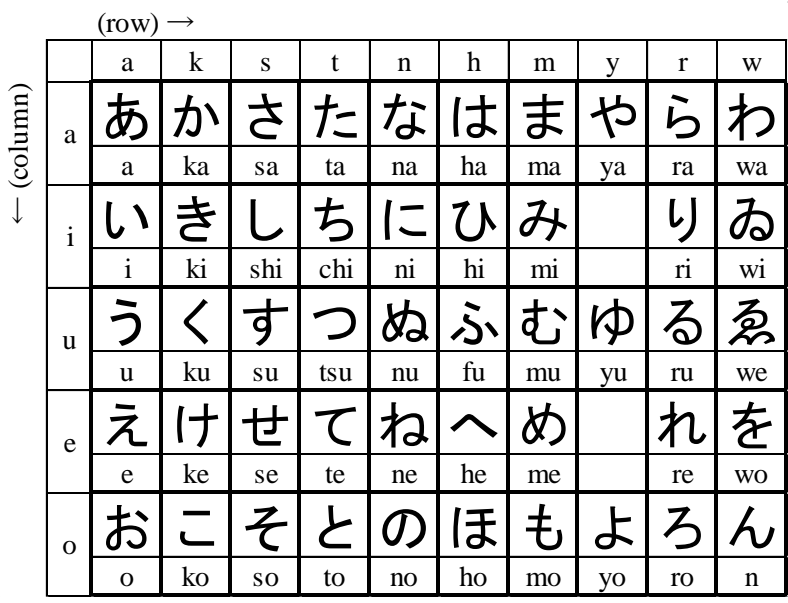

Fig. 6 Example of board for Hiragana characters

\section{B. Evaluation}

To quantitatively evaluate the operability of the device, its operation time was measured. The operation time was obtained by measuring the duration of the caregiver eye movement for entering a character using an eye movement measuring instrument (T.K.K. 2950, Takei Scientific Instruments) [8].

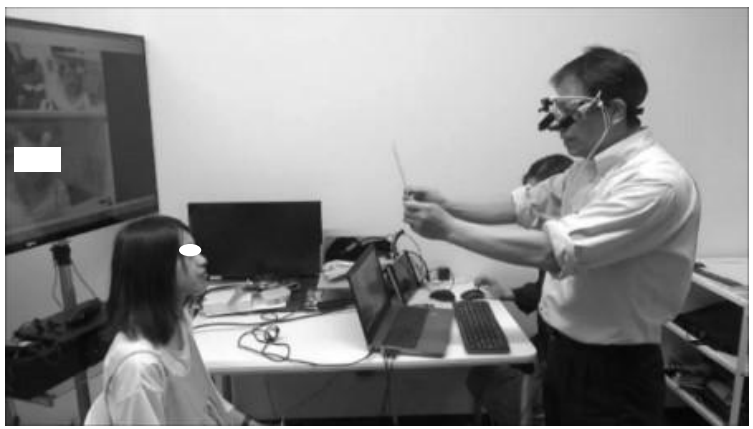

Fig. 7 Eye movement measurement with a transparent communication board viewed from the lateral side

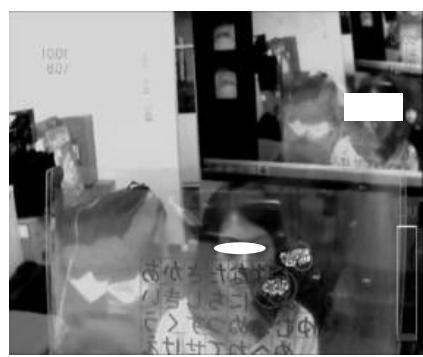

Fig. 8 Eye movement measurement with a transparent communication board viewed from the eye movement measurement device

Figure 7 showed the eye movement measurement with the transparent character board viewed from the lateral side, and Figure 8 showed the same measurement viewed from the eye movement measurement device. Figure 9 and 10 showed the same measurement with the tablets viewed from the lateral side and from the eye movement measurement device, respectively.

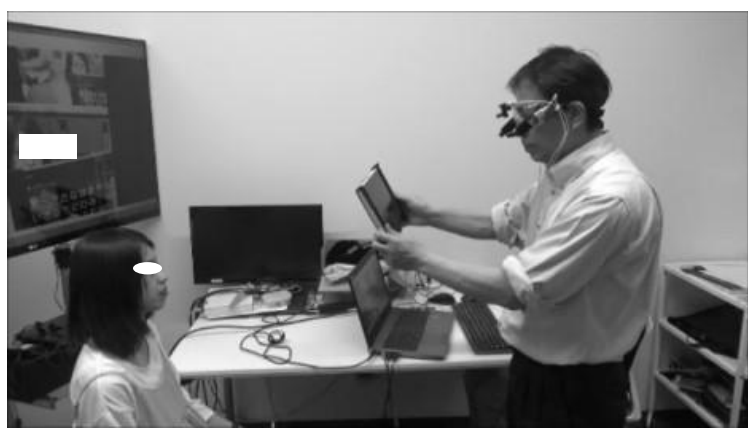

Fig. 9 Eye movement measurement with the tablets from the lateral side

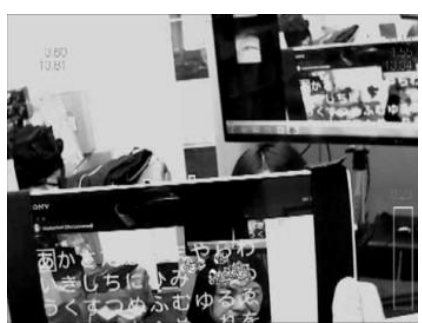

Fig. 10 Eye movement measurement with the tablet viewed from the eye movement measurement device

Since the process of arriving at the line and the column was different depending on each letter on the letter keyboard, the number of strokes $(\mathrm{N})$ was set for each character. For example, the number of strokes $(\mathrm{N})$ of "ki" was 4 because it was located on the " $k$ " row (with 2 rows) and on the " $i$ " column (with 2 columns); and the number of strokes of "nu" was 8 because it was located on the "n" row (with 5 rows) and on the "u" column (with 3 columns) (Figure 6).

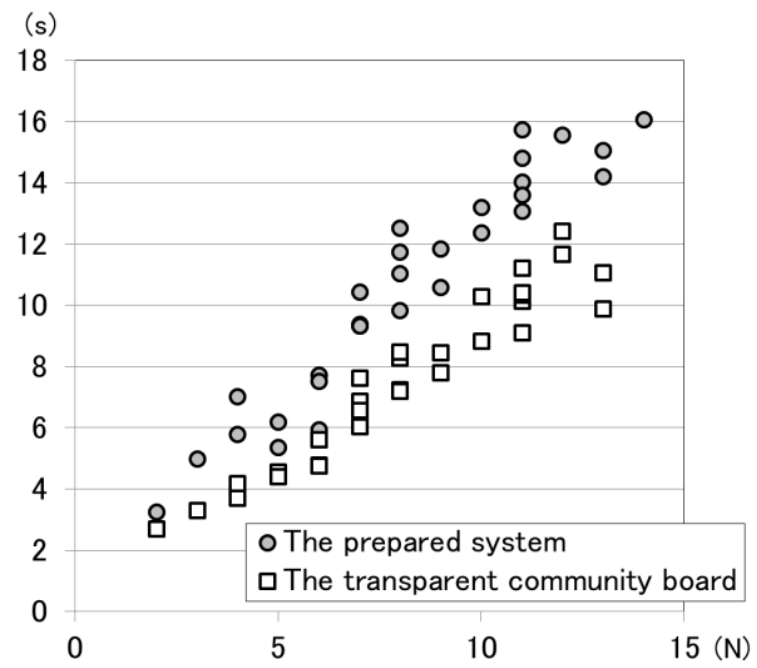

Fig. 11 Values of time of eye movement between the prepared system and the transparent communication board The subjects were two healthy volunteers (with one patient role and one caregiver role), and both subjects took turns to enter 10 character combinations, respectively, each 
consisting of three hiragana characters, which make no sense as Japanese words. The order of those 10 combinations was randomized. Each subject entered 15 characters (5 combinations) using a transparent communication board as well as the prepared device. The values of time (s) spent for eye movement with total 30 characters (for the total number of strokes) by the 2 subjects using the transparent communication board and the prepared device were plotted in the graph (figure 11). The average number of strokes was $8.17 \pm 3.04 \mathrm{~N}$. The average time of eye movement using the prepared device was $10.59 \pm 3.66 \mathrm{~s}$, while the average time thereof using the transparent communication board was 7.60 $\pm 2.74 \mathrm{~s}$.

\section{DISCUSSIONS}

The prepared device made it possible to enter characters without linearly aligning the visual line of the patient with that of the caregiver. In addition, it was not necessary to take notes because the entered characters were stored into the text input app. Hence, the burden on caregivers can be reduced.

On the other hand, the time for eye movement was significantly longer with the prepared device than the transparent communication board ( $\mathrm{p}<0.01$; Mann-Whitney test), and the time for eye movement became longer as the number of legs increased, and the variance became larger. This is probably because the video image of the patient is displayed later on the screen of the tablet for the caregiver, requiring an extended period to fix the locations of characters. It is expected that these negative impacts can be reduced by improving the communication speed of Wi-Fi connection and tablet performance.

\section{SUMMARY}

We developed an electronic transparent communication board to solve problems of the conventional transparent communication board and evaluated it.

Among the three problems of the conventional transparent communication board, the adjustment of the location of the transparent communication board seemed to become easier, because the device can be operated without aligning the visual line of the caregiver with that of the patient. However, the quantitative evaluation has not been conducted and will be done in the future. It took longer to operate the prepared device than the conventional transparent communication board, but it is expected that the negative impacts caused by the delays can be reduced, if the communication speed and tablet performance are improved. Characters were considered to become entered more easily with the prepared system than with the approaches depending on memories and notes. In this study, only a communication board for Hiragana characters was used, but communication boards for other characters including alphabets, numbers, and symbols are easily added on the app, so we will continue to evaluate the prepared system while adding communication boards.

We will keep striving to improve the system and continue to aiming at development of a communication assistance system easily useful for both patients and caregivers.

\section{ACKNOWLEDGMENT}

We thank the late Mr. Masanori Shoji who was a great pilot and engineer.

\section{REFERENCES}

[1] L. C. Wijesekera, P. N. Leigh, "Review Amyotrophic lateral sclerosis," Orphanet Journal of Rare Diseases, vol.4, 2009, pp.1-22.

[2] Neto LL, Constantini AC, Chun RYS. "Communication vulnerable in patients with Amyotrophic Lateral Sclerosis: A systematic review." NeuroRehabilitation, vol.40 (4), 2017, pp.561-568.

[3] Daisuke Yajima, Nguyen Xuan Son, Toyohiko Hayashi, Takashi Oda, Hiroaki Inui,"Development of a versatile control-interface Switch (VSN/1) using eye-blink for users with severe physical disabilities," biomechanisms, vol.23, 2016, pp.185-193.

[4] Ujwal Chaudhary, Bin Xia, Stefano Silvoni, Leonardo G. Cohen, Niels Birbaumer, "Brain-Computer Interface-Based Communication in the Completely Locked-In State," PLoS Biol. vol.15(1), 2017, e1002593.

[5] Takashi Nakajima, "Assistive technology for supporting communication for patients with incurable and progressive neuromuscular diseases, including transparent character boards, a mouth-shape character method, and an advanced Cybernic Interface device," J. Natl. Inst. Public Health, vol.66 (5), 2017, pp.491-496.

[6] Tomoya Miyasaka, Masanori, Shoji, Toshiaki Tanaka, "Development and Clinical Evaluation of an Eye Movement Input Device Designed to Assist Continuous Communication in Amyotrophic Lateral Sclerosis (ALS) Patients," J. Med. Biol. Eng., vol.31 (4), 2010, pp.265-271

[7] Tomoya Miyasaka, Toshiaki Tanaka, Takashi Izumi "Investigation of image-analysis detection limit for lifting of commissura laiorum," ACPT2013, I-P006, 2013.

[8] Shinya Mochiduki, Yuki Yokoyama, Keigo Sukegawa, Hiroki Sato, Miyuki Suganuma, Mitsuho Yamada, "Measurement of Accommodation and convergence eye movement when a display and 3D movie move in the depth direction simultaneously," IEICE Transactions on Fundamentals of Electronics, Communications and Computer Sciences, Vol.E101.A, Issue 2, 2018, pp.488-498. 